\title{
Mehrsprachigkeitstheater als nonverbale und mehrsprachige Kommunikation
}

\author{
Ursula Christine Bünger
}

\begin{abstract}
Zusammenfassung
Dieser Artikel verbindet das fremdsprachendidaktische Thema der Mehrsprachigkeit, das auch im Europäischen Referenzrahmen für das Lernen und Lehren für Sprachen vorgeschlagen wird, mit dem Theaterspielen (und somit affektiven und nonverbalen Kompetenzen). Zunächst wird die Theatertheorie von Mejerchol'd verknappt dargestellt und gezeigt, wie man deren Techniken für das Theaterspielen im Fremdsprachenunterricht verwenden kann. Nach der Vorstellung der kulturellen und psycholinguistischen Prämissen und Ziele der Mehrsprachigkeit erfolgt die Beschreibung eines kurzen Projekts, das im Rahmen einer nationalen Fortbildung in Italien im Fremdsprachenunterricht durchgeführt wurde. Abschließend wird das Feedback der Lernenden diskutiert und es werden mögliche Erweiterungen vorgeschlagen.
\end{abstract}

\section{Einführung}

Mein allgemeines Anliegen in der Theorie und Praxis der Didaktik Deutsch als Fremdsprache für Anfänger und fortgeschrittene Anfänger besteht darin, zu untersuchen, zu reflektieren und zu erproben, wie theaterpädagogische Elemente in die Diskussion um den regulären kompetenzorientierten schulischen Fremdsprachenunterricht in Europa eingegliedert werden können. In diesem Aufsatz geht es um die Verbindung des Ziels der Mehrsprachendidaktik (Europarat 2001) und des Theaterspielens. Theaterspielen lässt eine weitere „Sprache“ erlernen, und zwar die der nonverbalen Kommunikation von Emotionen. Diese Aspekte werden im - pragmatisch am Alltag und am Berufsleben orientierten - Referenzrahmen für das Lehren und Lernen von Sprachen in Europa (ibid.) etwas stiefmütterlich behandelt, wie schon Schmenk (2004) festgestellt hat.

Ich muss aus institutionellen Gründen in meinem Fremdsprachenunterricht Deutsch als Fremdsprache und meiner Reflektion darüber auch der Tatsache Rechnung tragen, dass europaweit eine kompetenzorientierte Didaktik eingesetzt wird. Diese meist kognitiv und verhaltensmäßig verstandenen „Kompetenzen“ versuche ich in meinem Unterricht um „humanistische“, also „affektive“ und „nonverbale“ Kompetenzen zu erweitern, denn meiner Ansicht 
nach sollte Fremdsprachenunterricht nicht nur am Endprodukt Test, sondern auch am Prozess, an Lernerbedürfnissen und an "Spaß“ für Lehrende und Lernende orientiert sein. Theaterpädagogik sollte sich andererseits den Anforderungen von fremdsprachlichen Kompetenzen und objektiven Bewertungen stellen.

An anderer Stelle (Bünger 2010) hatte ich beschrieben, wie KannBeschreibungen aus dem Europäischen Referenzrahmen (Europarat 2001) mit Theater als Methode mit Nullanfängern in der Schule im regulären Vormittagsunterricht realisiert werden können. Dabei ging es auch um die Auswirkungen des Filmens der fremdsprachlichen Szenen mittels Handyfilmkamera. Ich wollte zeigen, dass nicht nur affektive, sondern auch fremdsprachliche Kompetenzen durch Theaterspielen realisiert werden können, habe also Theaterspielen als Mittel zur Erreichung fremdsprachlicher Kompetenzen eingesetzt. Weiterhin wurde damit indirekt das Thema der „Neuen Medien“ im Fremdsprachenunterricht thematisiert und mit Theaterspielen in Verbindung gebracht. In jenem Projekt ging es mir also vorrangig um fremdsprachliche Kompetenzen, indem ich Handyfilmkamera u nd T heaterspielen a ls $\mathrm{M}$ ittel z ur Erreichung fremdsprachlicher Kompetenzen vorschlug.

In diesem Beitrag möchte ich hingegen zeigen, dass affektive Kompetenzen in mehreren Sprachen und non-verbal mit leicht fortgeschrittenen Anfängern (Niveau A1/A2) sprachlich und nicht-sprachlich realisiert werden können, behandle also fremdsprachliche, affektive und non-verbale Kompetenzen als Ziele gleichrangig. Durch die Verwendung von Theatertechniken wird somit das im Referenzrahmen enthaltene Konzept von Mehrsprachigkeit, das im zweiten Teil des Beitrags erörtert werden wird, um die affektive Komponente angereichert. Weiterhin geht es bei Theaterspielen nicht nur um Fremdsprachenerwerb, sondern auch um die Entwicklung non-verbaler Fähigkeiten. Ziel ist es, die Lernenden dafür zu sensibilisieren, dass es nicht nur Mutter- und Fremdsprache, sondern auch so etwas wie eine „affektive“ und „nonverbale“ Sprache oder Grammatik geben kann.

Theaterspielen als Ausdruck von Gefühlen durch Mimik, Gestik und Körperhaltung ist also hier nicht mehr „nur" Instrument zum Lernen der Fremdsprache, sondern auch ein eigenständiges Ziel. Somit verbinde ich das fremdsprachendidaktische Ziel der Mehrsprachigkeit mit nonverbalen und affektiven Zielen, die m.E. besonders gut mit Theaterspielen im Unterricht verbunden werden können.

Das vorliegende Projekt und die damit verbundenen bibliographischen Nachforschungen und Überlegungen entstanden während eines vom Europäischen Sozialfonds finanzierten Fortbildungskurses POSEIDON 2011 in Modica (RG), Sizilien. Ziel der nationalen Fortbildung war die interdisizplinäre Zusammenarbeit von Mutter-und Fremdsprachenunterricht, Zweitsprache Italienisch und den klassischen Sprachen Altgriechisch und Latein. Grundannahme der Didaktik der Mehrsprachigkeit ist, dass die Muttersprache für das Erlernen aller weiteren Sprachen eine Rolle spielt. Diese Wechselbeziehung wird anhand des Feedbacks der teilnehmenden Jugendlichen am Ende des Artikels kurz 
diskutiert werden.

\section{Theaterspielen in der Schule - warum?}

Theaterspielen ist also geeignet, den an den Rand gedrängten „humanistischen" Kompetenzen zu ihrem Recht zu verhelfen, auch da Emotionen und Körperausdruck im schulischen institutionellen Kontext selten zum Lerngegenstand gemacht werden (Galimberti 2007). Körper und Emotionen werden vor Büchern, Bildschirmen und Schulbänken geradezu ausgeblendet.

Nonverbale Kommunikation ist jedoch ein wichtiger Bestandteil von Kommunikation. Leider entwickelt die Schule diese Fähigkeiten kaum. Watzlawick bemerkte schon 1967, dass 90\% der Kommunikation nicht-sprachlich vor sich geht. Non-Verbales ist in der Alltagskommunikation also schon immer impliziert, bleibt meist jedoch unbewusst.

Der russische Regisseur Mejerchol'd (2012) hat den engen Zusammenhang zwischen Gedanken, Körperbewegung, Gefühl und Wort zum Gegenstand seines Biomechanik genannten Ansatzes gemacht. Da ich mit Lernenden ohne Theatervorkenntnisse arbeite (weder im rezeptiven noch im produktiven Sinn), verbinde ich diesen Ansatz mit der theaterpädagogischen Technik der „Statuen“ (Schewe 1993), lasse also die Körperbewegung „einfrieren“. Nach Mejerchol‘d ergibt sich die Emotion aus der Körperbewegung, welche wiederum das Wort „entstehen“ lässt.

Eine parallele Annahme dazu findet s ich in d er Affect Theory (Tomkins, 1962). Diese Theorie versucht, Gefühle in Kategorien einzuteilen und mit einer typischen beobachtbaren körperlichen Antwort zu verbinden (Freude, Lächeln).

Theaterspielen macht reale und „simulierte“ Interaktionen auch im Kontext von phantasievollen Szenarien möglich (Bünger 2010) und fördert darüber hinaus den Selbstausdruck und Empathie.

\section{Mehrsprachigkeit - was ist das?}

Im Folgenden stelle ich die Forschungsrichtungen zu Mehrsprachigkeit dar, da ich das Theaterprojekt in diesen mehrsprachlichen Kontext gestellt habe.

Der Referenzrahmen (2001: 163ff) unterstreicht ausdrücklich die Bedeutung der Mehrsprachigkeit für Europa. Im Kontext dieses für Europa bedeutenden Handbuchs ist mit Mehrsprachigkeit gemeint, dass jeder Bürger außer der Muttersprache auch zwei weitere Nationalsprachen Europas beherrschen sollte. Im Referenzrahmen selbst wird außer den vier Fertigkeiten dem Aspekt der Sprachmittlung Bedeutung zugemessen. Sprachmittlung ist als Vermittlung definiert, $\mathrm{d}$ ie $\mathrm{z}$ wischen e inzelnen $\mathrm{S}$ prachen, $\mathrm{i}$ nnerhalb e iner $\mathrm{S}$ prache und innerhalb von semiotischen Systemen vermittelt (hier: Sprache, Fremdsprache und Körpersprache) (Dell'Ascenza/ Curci, s.d.). Dies bedeutet, dass die Muttersprache der Lernenden wieder eine Rolle spielt, dass also wieder 
übersetzt wird, was bis jetzt nach dem kommunikativen Ansatz in der Fremdsprachendidaktik ausgeblendet wurde. ${ }^{1}$

Innerhalb der Forschung zur Mehrsprachigkeit finden sich zwei Forschungsstränge (Bausch / Königs / Krumm 2004). Der erste Forschungsstrang ist psycholinguistischer Natur und erforscht die psycholinguistische und kognitive Repräsentation von Fremdsprachen. Hu definiert b eispielsweise individuelle Mehrsprachigkeit als Kompetenz, „bei der mehrere Sprachen im mentalen System einer Person interagieren und miteinander vernetzt sind (2010: 214)". Aus psycholinguistischer Sicht haben beispielsweise Studien ergeben, dass einerseits eher Muttersprache und früh erlernte Fremdsprachen und andererseits spät erlernte Fremdsprachen eher miteinander vernetzt sind (Hufeisen 2004: 23).

Die zweite Forschungsrichtung siedelt Mehrsprachigkeit als soziokulturelle und interkulturelle Theorie und Praxis an und bezeichnet Sprachen als Interaktionen innerhalb eines Kontextes und einer Kultur. Daher beschäftigt sich dieser Ansatz auch mit migrationsbedingten Lernersprachen (Hu 2003). In Italien bezieht der Ansatz daher auch die Muttersprache Italienisch und Italienisch als Zweitsprache mit ein, sowie die im italienischen Kontext nicht als Fremdsprachen betrachteten und unterrichteten klassischen Sprachen Latein und Altgriechisch. Ich beziehe mich zwar auf diesen Ansatz, beziehe aber auch Ergebnisse aus der psycholinguistischen Forschungsrichtung mit ein. In diesem Projekt werden die Muttersprache der Lernenden Italienisch, sowie Englisch und Deutsch als Fremdsprachen (sowie Deutsch als Muttersprache der Autorin und Lehrenden) eingesetzt. ${ }^{2}$

Kognitionspsychologisch wird mehrsprachliches Vorgehen mit der Interaktion von Vorwissen mit neuem Wissen, sowie der Bewusstmachung von Differenz und Verbindungen zwischen Sprachen und von Lernstrategien begründet. Mein Projekt zielt jedoch darüber hinaus darauf ab, durch Theaterspielen im Bereich der Emotionen den Lernenden den Stellenwert von affektiven und nonverbalen „Sprachen" klar zu machen und zu entwickeln.

Nun wende ich mich der Frage zu, was die fremdsprachendidaktische Forschung zur Mehrsprachigkeit zum Thema Körpersprache und Emotionen beiträgt. Die Frage, ob sich verschiedene Fremdsprachen auch verschieden auf der körpersprachlichen Ebene ausdrücken, muss leider ausgeklammert werden, da mir kein Beitrag aus der Fremdsprachendidaktik dazu bekannt ist. Wie sieht es mit Emotionen und Mehrsprachigkeit aus?

Kramsch (1993) fokussiert auf die Bedeutung der Sprache für die eigene Identität und somit auf die emotionale Valenz. Jeder (auch teilweise) Spracherwerb verändert unsere Identität. Nach diesem Modell werden auch nichtkompetente Sprecher einer Sprache akzeptiert, solange sie Sprache und

\footnotetext{
${ }^{1}$ Die Begriffe „Muttersprache“ und „Nationalsprache“ könnten in einem immer mehr multikulturell, globalisiert und bilingual werdenden Europa kritisch gesehen werden, da Fremdsprachen inzwischen die nationalen Grenzen überschreiten (vgl. Bünger et.al. 2007). Da sich der Referenzrahmen jedoch auf Nationalsprachen bezieht, werde ich den Begriff weiter benutzen.

${ }^{2}$ Wäre eine Migrantin in der Lernergruppe gewesen, hätte ich auch deren Muttersprache mit eingesetzt.
} 
Sprachmischungen funktionell einsetzen.

Die emotionale Valenz der verschiedenen Sprachen bei Kindern wurde vor allem auch von Krumm (Oomen-Welke; Krumm 2004: 7) untersucht. Hufeisen (2004: 21) weist dagegen auf die Bedeutung von positiven und negativen Lernvorerfahrungen hin $^{3}$.

\section{Theaterspielen im Bereich der Mehrsprachigkeit - warum?}

Theaterspielen ist besonders geeignet, die fremdsprachendidaktische Arbeit im Rahmen der Mehrsprachigkeit anzureichern, da dieser Ansatz reale Interaktionen und authentischen fremdsprachlichen und nonverbalen Ausdruck fördert, vor allem da im Rahmen des vorliegenden Projekts die affektive und nonverbale oder mimische, gestuelle und posturale Ebene der Verständigung ebenfalls als „Fremd-Sprache“ im Bereich der Mehrsprachigkeit angesehen wird. Der Fokus richtet sich auf die Entwicklung der „Körpersprache“ (Mimik, Gestik, Körperhaltung) und von Emotionen als weiteren „Fremd-Sprachen“ in Ausdruck und Interaktion.

Theaterspielen erscheint mir als eine Möglichkeit, einen Mangel an Ausdruck, Interaktion und empathischer Reaktion zu überwinden und fremdsprachliches und non-verbales Ausdrücken, Interagieren und Reagieren auf den Anderen einzuüben, also „humanistische“ Kompetenzen zu entwickeln. Ziel des hier kurz skizzierten Projekts ist also nicht nur die fremdsprachliche Mehrsprachigkeit, sondern auch das zumindest ansatzweise Erlernen einer anderen, nonverbalen Umgangsform und somit „Sprache“ im schulischen und täglichen Kontext.

\section{Meine fremdsprachlichen Ziele}

Meine Lernenden haben als Muttersprache alle Italienisch und lernen seit der Grundschule Englisch. Auf der Sekundarstufe, an der ich unterrichte, kommen die Fremdsprachen Französisch, Latein und Deutsch hinzu.

Für den Unterricht Deutsch als Fremdsprache benutze ich die KannBeschreibung „Kann Gefühle ausdrücken und darauf reagieren (Europarat 2001: 80)" in Verbindung mit der Sprachhandlung des Motivierens von Gefühlen durch Handlungen und Situationen in der Vergangenheit. Aus grammatischer Sicht übe ich so die Vergangenheitsform in den eingesetzten Sprachen, aus lexikalischer Sicht setze ich die Konnektoren „denn“ mit Hauptsatz und fakultativ „weil“ mit Nebensatz ein. Dies kann natürlich entsprechend dem Lernstand der eigenen Lerngruppe variiert werden. Das Theaterspielen gibt dazu also einen konkreten Kontext (zum Begriff des Kontexts in der

\footnotetext{
${ }^{3}$ Beliebt ist in der sog. „Tertiärsprachendidaktik“ für den Bereich Deutsch als Fremdsprache ja der Rückbezug auf Englisch, da es lexikale und syntaktische Gemeinsamkeiten zwischen diesen ursprünglich miteinander verwandten Sprachen gibt. Ungeklärt bleibt, was passiert, wenn die Lernenden negative Lernvorerfahrungen mit dieser Sprache gemacht haben.
} 
Fremdsprachendidaktik, vgl. Bünger 2010), in dem die Verwendung der Konnektoren „denn“ und „weil“ nicht mehr eine „staubtrockene“ Einsetzübung darstellen, sondern einen affektiven Sinn erhalten. ${ }^{4}$

Diese Vorgehensweise wird in mehreren Fremdsprachen durchgeführt, und zwar mündlich (Produktion). Dabei müssen die Sätze nicht metalinguistisch analysiert werden, sondern können als unanalysierte chunks (Bünger 2010; Wettemann 2007) eingesetzt werden. Sieht die Unterrichtsplanung auch die metalinguistische Analyse vor, empfehle ich den Einsatz entweder als warmingup am Anfang oder zum Abschluss des Unterrichtsteils zur metalinguistischen Arbeit.

In der Muttersprache (Italienisch) wird dagegen schriftlich ein Tagebuch verfasst, in dem in einem inneren Monolog beschrieben wird, wie es zu dem dargestellten und in der Fremdsprache ausgedrückten Gefühl kam. Diese veränderte Textform war nicht geplant und ergab sich aus dem Prozess der Theaterarbeit im Fremdsprachenunterricht. Die Umformung eines theatralisch dargestellten Satzes in der Fremdsprache zu einer anderen Textsorte in der Muttersprache stellt eine Technik aus der Sprachmitttlung dar. Dabei wird von einer Textsorte in die andere Textsorte übertragen bzw. „übersetzt“ (Dell'Ascenza/ Curci, s.d.).

Im Folgenden soll nun das Projekt beschrieben werden, das ich im Oktober 2011 in einer zweiten Klasse eines Liceo Linguistico (neusprachlichen Gymnasiums) durchgeführt habe, und zwar, mit ca. fünfzehnjährigen Jugendlichen, die Deutsch, Französisch und Latein als zweite Fremdsprache nach Englisch im zweiten Jahr lernen und sich damit fremdsprachlich auf dem Kompetenzniveau A1/A2 befinden. Aus Zeitgründen habe ich diesem Projekt nur eine knappe Stundenzahl widmen können, weswegen ich auch Erweiterungsvorschläge skizziere. Abschließend wird die Selbstevaluierung der Lernenden im Gruppenfeedback dargestellt und diskutiert.

\section{Mein Projekt}

Ein bedeutendes Ziel des Projekts ist auch die Zusammenarbeit zwischen den einzelnen Disziplinen bzw. Fremdsprachen. So erklärten sich meine Italienischund Englischkollegin bereit, zeitgleich zu meinem Projekt Vokabeln zum Thema Emotionen und Konnektoren in der Muttersprache Italienisch und in der ersten Fremdsprache Englisch zu wiederholen. In meinem Projekt habe ich also mit den Fremdsprachen Deutsch und Englisch und der Muttersprache Italienisch im Deutschunterricht gearbeitet.

\footnotetext{
${ }^{4}$ Durch die anschließend mögliche Erweiterung des Ausdrucks von Fremdsprache und Körpersprache kann diese mündliche Produktion zur mündlichen Interaktion und zum empathischen Dialog werden, wie in den Erweiterungen am Schluss dargestellt wird.
} 


\subsection{Erste Stunde}

Ziele: Die Lernenden sollen auf das Thema eingestimmt werden. Die Lexik soll wiederholt und erweitert und die kausalen Konnektoren „weil“ und „denn“ sollen wiederholt werden.

Ich stelle den Lernenden meine Ziele und das Projekt vor. Danach beginnt der erste fremdsprachliche Teil: die Lernenden schreiben schon bekannte Vokabeln zum Thema Emotionen auf Deutsch und auf Englisch an die Tafel und ins Heft, danach erfolgt eine Wortschatzerweiterung durch ein Assoziogramm. Auch dieser Teil dient der Einstimmung auf das Thema und der Wiederholung von Vokabeln. Im zweiten Teil der ersten Stunde werden die kausalen Konnektoren vorgestellt bzw. wiederholt und mündlich eingeübt. Ich beschließe, mich auf den Hauptsatz zu beschränken und „weil" mit Nebensatz nicht einzuführen, um die grammatischen Elemente auf das Minimum zu reduzieren.

Danach frage ich die Lernenden in der Muttersprache, wie sie sich emotional in Bezug auf eine durchgeführte Klassenfahrt fühlen und bitte sie, ihre Emotion auf Deutsch zu nennen und zu begründen. Eine Lernerin führt zwei gegensätzliche Emotionen an, und ich greife das in der Muttersprache auf: Emotionen sind nicht immer eindeutig, sondern auch ambivalent.

Als Hausaufgabe schreiben die Lernenden einfache Sätze in den Fremdsprachen Deutsch und Englisch ins Heft, indem sie denen eine (oder zwei) Emotion(en) mit einer situativen Begründung in der Vergangenheit oder im Präsens verbinden.

\subsection{Zweite und dritte Stunde}

Ziele: Aus verbaler Sicht wird der adversative Konnektor "trotzdem" eingeführt. Es beginnt die nonverbale Arbeit, indem die Lernenden Elemente non-verbaler Sprache kennenlernen und Emotionen darstellen.

Die Lernenden lesen die Sätze laut aus dem Heft vor. Danach ergänzen sie ihre Sätze mit einem weiteren Satz, der mit ,trotzdem“ beginnt, um der Ambivalenz von Emotionen gerecht zu werden. Ich weise darauf hin, dass nach „denn“ keine Inversion erfolgt, nach ,trotzdem“ aber Inversion erfolgt. Danach beginnt der „theatralische“ Teil. Die Lernenden stellen sich einzeln vor die Klasse und stellen eine Emotion mit der Mimik, mit der Gestik und mit der Körperhaltung dar. In dieser Phase arbeite ich mit der Technik der Statuen (Schewe 1993).

Als Anreiz spreche ich die Emotion in der Muttersprache, auf Deutsch, und auf Englisch aus (im Zweifelsfall in der Muttersprache), und die Lernenden drücken die Emotion nonverbal aus. Ich weise darauf hin, dass die Körperhaltung bzw. die Geste die Emotion hervorruft.

Als Hausaufgabe schreiben die Lernenden mindestens fünf Sätze in der Muttersprache als „Tagebuch“ oder „inneren Monolog“ zu einer Emotion in ihr Heft oder auf ein Blatt Papier. Damit wird die „Übersetzung“ in die Muttersprache und in eine andere Textsorte eingeleitet, und nonverbale und verbale Elemente werden zusammengeführt. 


\subsection{Vierte und fünfte Stunde}

Ziele: Einzelne Sätze, die Emotionen ausdrücken und begründen, werden in den Fremdsprachen und in der Muttersprache auswendig gelernt. Die verbalen Elemente sollen mit nonverbalen Elementen verbunden werden. Damit wird die „Aufführung“ mehrsprachig und verbal vorbereitet.

Die Lernenden wählen sieben Emotionen aus. Diese Emotionen werden mit einer konkreten Situation, die die Emotion hervorgerufen hat, begründet. Dies wiederholen sie zunächst sitzend in den Sprachen Italienisch, Deutsch und Englisch. Dabei kann auch ein expliziter Sprachvergleich stattfinden. Danach stellen sie sich in zwei Gruppen im Raum gegenüber und üben a) die Sätze in den drei Fremdsprachen; dann b) darstellend als „eingefrorene" Statuen (zu der Theatertechnik der "Statuen“ in der Gruppe Schewe 1993), indem sie einen Schritt vorwärts gehen und noch ohne Sprache; dann c) gehen sie einen Schritt vor, nehmen die Haltung ein, sehen sich an und sprechen die Sätze. Ich gebe dabei den sprachlichen und nichtsprachlichen Rhythmus durch Fingerschnipsen vor. ${ }^{5}$

In dieser Phase beschließen die Lernenden mit meinem Einverständnis, das Italienische - die Muttersprache - wegzulassen, weil es ihnen „komisch“ vorkommt. Diese Reaktion ist als interessant zu bewerten; es scheint so, als ob die "fiktive“ Situation des Theaterspielens nur die „fiktive“ oder „künstliche“ Fremdsprache zulässt, nicht aber die Muttersprache (vgl. Diskussion weiter unten).

Zum Abschluss „frieren“ die Lernenden ihre jeweiligen Sätze ein, und führen - ohne sich zu bewegen - einen Dialog mit mir als „teacher-in-role“ (Schewe 1993). Aus der individuellen „Aufführung“ wird ein Gruppenerlebnis.

\subsection{Sechste und siebte Stunde}

Ziel: Es soll eine Generalprobe für die filmische Aufnahme simuliert werden, was das „Einschleifen“ der Sätze zur Folge hat. Anschließend wird die Szene gefilmt.

Die Gruppe stellt sich in eine einzige Reihe und probt noch einmal die aufeinander folgenden Sätze in den Sprachen Deutsch und Englisch. Danach wird der „innere Monolog“ in der Muttersprache dazu genommen: auf mein jeweiliges Fingerschnipsen (Begründung: siehe oben) hin

- kommt eine Lernende in die Mitte mit dem Blatt Papier, auf dem der „innere Monolog“ leserlich zu der jeweiligen Emotion steht, liest den Monolog vor, legt das Blatt ab und geht in die Reihe zurück;

- entspannt sich die Gruppe

\footnotetext{
${ }^{5}$ Ich ging davon aus, dass die Lernenden in der kurzen Zeit des Projekts noch kein Gefühl für den Rhythmus von Fremdsprache entwickeln könnten.
} 
- spannen die Lernenden die Muskeln und den Körpertonus und die Aufmerksamkeit an, während ein Punkt hinter der Wand des Klassenzimmers fixiert wird;

- geht die Gruppe einen Schritt mit dem rechten Fuß vor;

- nimmt die Gruppe eine Körperhaltung ein, die die Emotion ausdrückt. Dabei soll auch auf Gestik und Mimik geachtet werden;

- spricht den Satz auf Deutsch;

- spricht den Satz auf Englisch;

- hält die Körperspannung.

In der siebten Stunde wurde die Szene mit dem Handy einer Lernenden aufgenommen (zum Vorgehen vgl. Bünger 2010).

\section{Lernerfeedback und Diskussion}

Interessant war für mich die Bemerkung während der Theaterarbeit, die ja aus der Planungsphase vorsah, denselben Satz in der Muttersprache und in den zwei Fremdsprachen auszudrücken, dass die Jugendlichen das Aussprechen des Satzes in der Muttersprache als „lächerlich“ empfanden und ablehnten. Aus diesem Grunde habe ich das Projekt verändert und habe die Jugendlichen gebeten, in der Muttersprache einen „inneren Monolog“"zu schreiben. Ich führe das auf den „foreign language mode“ (Hufeisen 2004) zurück. Das bedeutet, dass entweder die Fremdsprachen allgemein oder die dritten, also alle nach der L2 gelernten Fremdsprachen neurokognitiv eher miteinander vernetzt sind.

Es könnte allerdings auch sein, dass nicht derselbe Satz in Übersetzung zur Aufführung kommen muss, sondern dass schlicht das entsprechende Kompetenzniveau in den verschiedenen Sprachen berücksichtigt werden müsste. Dann könnte im Deutschen ein Satz auf dem Kompetenzniveau A2, im Englischen könnten dagegen mehrere Sätze auf dem Kompetenzniveau B1/B2 gespielt und verbalisiert werden.

In der anschließenden Stunde bat ich die Lernenden um ein Feedback zum Projekt. Überraschender- und erfreulicherweise für mich kam spontan die Feststellung, sie hätten die Bedeutung der nonverbalen Sprache erlernt: „Wir haben gelernt, dass auch der Körper spricht“. Dieses Ziel wurde also erreicht.

Zur Mehrsprachigkeit war die Bemerkung zu hören, sie seien „confusi“ (verwirrt). Eine weitere Erklärung war den Lernenden nicht zu entlocken. Dies bedeutet, dass die didaktische Literatur sich noch mehr Gedanken machen muss zum gleichzeitigen Erlernen schulischer Fremdsprachen: wie geht man vor und wie verständigt man sich interdisziplinär, damit das gleichzeitige Erlernen und Benutzen von zwei Sprachen nicht zu dem „diffusen Gefühl“ der Verwirrung führt, das meine Lerner zum Ausdruck brachten. Es könnte auch sein, dass es 
die „Neuheit" des Projekts war, die diese Reaktion hervorbrachte. Ich würde also in einem weiteren Projekt erklären, dass es um dritte Fremdsprachen geht und warum.

Ein weiteres Problem ist das der Leistungsmessung, das ein grundsätzliches Problem bei kreativer Unterrichtsgestaltung (z.B. bei freiem oder kreativem Schreiben) darstellt, und über das bezüglich des Theaterspielens in der Fremdsprache nachgedacht werden müsste, vor allem innerhalb eines Kompetenzmodells, das ja von der Leistungsmessung ausgeht. Kann ich einem Lernenden eine bessere Note geben, weil er sich besser körperlich ausdrücken kann oder weil er in der Lage ist, eine Emotion angemessen nonverbal und verbal auszudrücken? Dazu ist anzumerken, dass oft gerade so genannte „leistungsschwächere'“ Lernende viel Spaß am und Talent zum Theaterspielen zeigen, während es „leistungsstärkere“ Lernende gibt, die sich manchmal nicht einmal dazu imstande sehen, es auszuprobieren. Wie im letzten Beitrag (Bünger 2010) angemerkt, „zwinge“ ich meine Lernenden grundsätzlich nicht dazu, Theater zu spielen oder auch nur theaterpädagogische Elemente wie das mit verschiedenen Emotionen vorgetragene und so verfremdende Dialoge oder Sätze auszuprobieren. Außerdem ist auch die Bewertung von Mehrsprachigkeit ein offenes Problem.

Da es sich bei meinem Unterrichtsvorschlag um ein zeitlich sehr begrenztes Modell handelt, möchte ich im Folgenden noch Ideen zur Erweiterung andeuten:

\subsection{Erste Erweiterung: Emoticons mit einbeziehen}

Ziel: Emoticons sollen nonverbal und verbal in den verschiedenen Fremdsprachen dargestellt werden.

Es könnte zum Thema gemacht werden, welche Emoticons von den Lernenden häufig benutzt werden, was sie ausdrücken und wie das in den Fremdsprachen sprachlich umgesetzt wird. In einem zweiten Schritt versuchen die Lernenden, die Emoticons mimisch umzusetzen. Darüberhinausgehend kann der ganze Körper mit einbezogen werden, um die Emotion in der ganzen Körperhaltung darzustellen.

\subsection{Zweite Erweiterung: „Drittsprachen“}

Ziel: Die erste Fremdsprache (Englisch) soll ausgeklammert werden.

Für ein weiteres Projekt würde ich nur die Tertiärsprachen Französisch und Latein in den Deutschunterricht der Lernenden einbinden, da aus psycholinguistischer Sicht die Zweitsprachen (hier: alle Sprachen nach der ersten Fremdsprache Englisch) kognitiv miteinander vernetzt sind.

\subsection{Dritte Erweiterung: migrationsbedingte Lernersprachen}

Ziel: Migrationsbedingte Lernersprachen sollen in den Fremdsprachenunterricht mit einbezogen werden. 
Im Rahmen der Migrationsbewegungen innerhalb von Europa sind immer häufiger zwei- bzw. mehrsprachige Lernende im schulischen Fremdsprachenunterricht anzutreffen. Daher könnten also auch Lernende mit Migrationshintergrund, soweit in der Lerngruppe präsent, mit ihrer Muttersprache in das sprachliche Geschehen mit eingegliedert werden. Weiterhin wäre es natürlich auch wünschenswert, klassische Sprachen wie Latein mit einzubeziehen.

Für das vorliegende Theaterprojekt bedeutet dies, dass auch migrationsbedingte Lernersprachen eine Rolle spielen. Wie die Sprachbiographie im Europäischen Sprachenportfolio (Europarat 2001) hat dieses Vorgehen auch einen pädagogischen Wert, nämlich den Ursprungssprachen der Lernenden Wert beizumessen. Wenn im hier beschriebenen Projekt eine Migrantin oder ein Migrant in der Lernergruppe gewesen wäre, hätte ich also das Projekt außer in Italienisch, Deutsch und Englisch auch in seiner Muttersprache durchgeführt.

\subsection{Vierte Erweiterung (im Anschluss an das dargestellte Projekt)}

Ziel: Die einzelnen Sätze sollen zu verbalen und nicht-verbalen Interaktionen erweitert werden.

Nach der eben dargestellten Sequenz für die einzelnen Emotionen, die an eine bestimmte Situation gebunden werden, die die Emotion hervorgerufen hat, und die zusammen mit den Lernenden entschieden wurden, entspannen sich die Lernenden. Danach - auf ein weiteres Fingerschnipsen - nehmen sie wieder die „gespannte“ Körperhaltung an. Die Lehrende fragt nun (entweder auf Deutsch oder auf Englisch) einzelne Lernende: „Was hast du?" / „What's the matter?"Der oder die Angesprochene antwortet in der jeweiligen Fremdsprache, indem er auch die entsprechende Haltung, Mimik und Gestik einnimmt. Die Lehrperson nähert sich der Lernenden und reagiert nur nonverbal (beispielsweise kann nach dem Satz, „ich bin traurig, denn mein Hund ist gestorben“ tröstend eine Hand auf die Schulter gelegt werden). Dieselbe Sequenz kann unter den Lernenden selbst vor sich gehen.

\section{Schlussbetrachtung}

Ich ziehe aus meinem Projekt folgende zwei Schlussfolgerungen für eine didaktisch begründete Weiterarbeit: Erstens scheint Theaterspielen wirklich dazu geeignet zu sein, Jugendlichen die Bedeutung nonverbaler Kommunikation klar zu machen, wie aus dem einhelligen Feedback dazu hervorgeht.

Zweitens ist jedoch die Stellung der Muttersprache zu den Fremdsprachen (hier: Deutsch und Englisch) noch näher zu untersuchen. Für das italienische Projekt wurde davon ausgegangen, dass die Muttersprache für das Erlernen der Zweitsprachen eine Rolle spielt. Hufeisen dagegen nimmt an, dass die Zweitsprachen mental zusammen repräsentiert und von der Muttersprache getrennt sind. Dieses Dilemma wäre zu klären, denn die didaktischen Folgerungen aus diesen beiden Prämissen wären natürlich völlig verschieden. 
Ich hoffe daher, dass sich die Forschung in der Mehrsprachigkeitsdidaktik dieser Frage in der Zukunft vermehrt annimmt.

Literatur -

Baumann, Zygmunt (2002; 2010): Modernità liquida. Bari: Laterza

Bausch, Karl Richard; Königs, Frank \& Krumm, Hans-Jürgen (Hrsg.) (2004): Mehrsprachigkeit im Fokus: Arbeitspapiere der 24. Frühjahrskonferenz zur Erforschung des Fremdsprachenunterrichts. Tübingen: Narr

Bünger, Ursula Christine (1999): La 'drammapedagogia' nell'insegnamento delle lingue straniere. In: Quaderni del Lend, Milano, dicembre 1999, anno XXVIII 5, 18-24

Bünger Ursula Christine; Curci, Annamaria (2007): Intervista a Claire Kramsch, Università di Berkeley In: Quaderni del Lend, Milano, giugno 2007, anno XXXVI 3, 21-49

Bünger, Ursula Christine (2010): Individualisierung und Fiktionalisierung der Kann-Beschreibungen des Europäischen Referenzrahmens durch Dramapädagogik mit Handyfilmen. In: Scenario II/02, 41-61

Europarat (2001): Gemeinsamer europäischer Referenzrahmen für Sprachen lernen, lehren, beurteilen. Berlin u.a.: Langenscheidt

Dell'Ascenza, Carmen; Curci, Annamaria (s.d.): La didattica della mediazione linguistica. http://risorsedocentipon.indire.it/offerta_formativa/c/index.php?action=copert (27.10.2012)

Galimberti, Umberto (2007): L'ospite inquietante. Il nichilismo e i giovani. Milano: Feltrinelli

Hu, Adelheid (2003): Schulischer Fremdsprachenunterricht und migrationsbedingte Mehrsprachigkeit. Tübingen: Narr

Hu, Adelheid (2010): Einträge zu „Mehrsprachigkeit“ und Mehrsprachigkeitsdidaktik.“ In: Metzler Lexikon Fremdsprachendidaktik, 214-217

Hufeisen, Britta; Neuner, Gerhard (2003) (Hrsg.): Mehrsprachigkeitskonzept, Tertiärsprachen, Deutsch nach Englisch. Strasbourg: European Center for Modern Languages

Hufeisen, Britta (2004): Deutsch und die anderen Fremdsprachen im Kopf. In: Fremdsprache Deutsch 31, 19-23

Kramsch, Claire (1993): Context and Culture in Language Teaching. Oxford: Oxford University Press 
Krumm, Hans-Jürgen (2008): Gemeinsamer europäischer Referenzrahmen für Sprachen: Ziele, Wirkungen und Nebenwirkungen. In: Fremdsprache Deutsch 38, 26-28

Mejerchol'd, Vsevolod (a cura di Malcovati Fausto): (1993) L'attore biomeccanico. Milano: Ubulibri

Oomen-Welke, Ingeborg; Krumm, Hans-Jürgen (2004): Sprachenvielfalt eine Chance für den Deutschunterricht. In: Fremdsprache Deutsch 31, $5-13$

Schewe, Manfred (1993): Fremdsprache inszenieren. Zur Fundierung einer dramapädagogischen Lehr- und Lernpraxis. Oldenburg: Zentrum für pädagogische Berufspraxis der Carl-von-Ossietzky Universität Oldenburg

Schmenk, Barbara (2004): Drama in the Margins? The Common European Framework of Reference and its Implications for Drama Pedagogy in the Foreign Language Classroom. In: German as a Foreign Language (GFL-Journal) 1, 7-23 [http://www.gfl-journal.de/1-2004/schmenk.pdf (30/01/2011)]

Tomkins, Sylvan S. (1962): Affect Imagery Consciousness: The Positive Affects (Vol. 1). Springer: New York

Watzlawick, Paul; Beavin, Janet Helmick \& Jackson, Don D. (1967): Pragmatica della comunicazione umana. Roma: Astrolabio

Wettemann, Ulrich (2007): Kein trockener Anfängerunterricht mehr! Eine dramapädagogische Modelleinheit für die ersten DaF Stunden. In: Scenario I/01, 70-97 